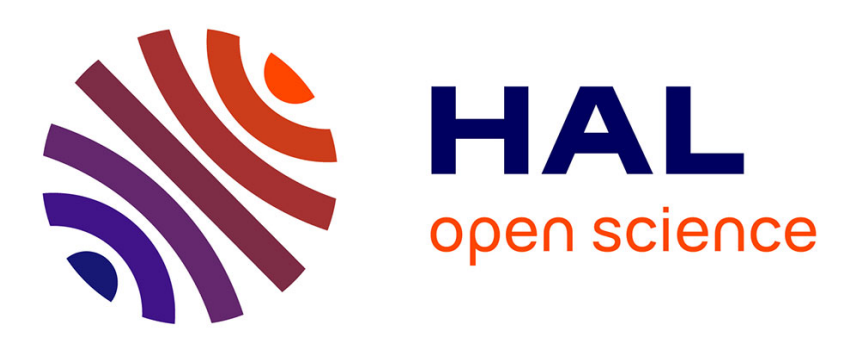

\title{
Error estimation and adaptive mesh refinement for the unstructured inductive PEEC formulation
}

Kouceila Alkama, Gerard Meunier, Olivier Chadebec, Jean-Michel Guichon, Bertrand Bannwarth, Enrico Vialardi, Jonathan Siau, Remy Perrin-Bit

\section{- To cite this version:}

Kouceila Alkama, Gerard Meunier, Olivier Chadebec, Jean-Michel Guichon, Bertrand Bannwarth, et al. Error estimation and adaptive mesh refinement for the unstructured inductive PEEC formulation. IEEE Transactions on Magnetics, 2022, 58 (3), 10.1109/TMAG.2021.3137657 . hal-03502851

\section{HAL Id: hal-03502851 https://hal.science/hal-03502851}

Submitted on 26 Dec 2021

HAL is a multi-disciplinary open access archive for the deposit and dissemination of scientific research documents, whether they are published or not. The documents may come from teaching and research institutions in France or abroad, or from public or private research centers.
L'archive ouverte pluridisciplinaire $\mathbf{H A L}$, est destinée au dépôt et à la diffusion de documents scientifiques de niveau recherche, publiés ou non, émanant des établissements d'enseignement et de recherche français ou étrangers, des laboratoires publics ou privés. 


\title{
ERROR ESTIMATION AND ADAPTIVE MESH REFINEMENT FOR THE UNSTRUCTURED INDUCTIVE PEEC FORMULATION
}

\author{
Kouceila Alkama ${ }^{1,2}$, Gérard Meunier ${ }^{1}$, Olivier Chadebec ${ }^{1}$, Jean-Michel Guichon ${ }^{1}$, Bertrand Bannwarth ${ }^{1}$, \\ Enrico Vialardi², Jonathan Siau ${ }^{2}$, Rémy Perrin-Bit ${ }^{2}$ \\ ${ }^{1}$ Univ. Grenoble Alpes, CNRS, Grenoble INP, G2Elab, 38000 Grenoble, France \\ ${ }^{2}$ Altair Engineering France, 38240 Meylan, France
}

\begin{abstract}
An adaptive mesh refinement procedure is presented in order to address efficiently low frequencies electromagnetic problems with the unstructured PEEC method. An a posteriori error estimator based on an equilibrated energy criterion is proposed, which means that two dual solutions are necessary. Actually, knowing that there is no dual formulation for the PEEC method, an auxiliary method is proposed in order to calculate a second admissible solution. This method is based on a projection of the PEEC solution from facets to edges function space in order to ensures the continuity of the electric field in the mesh. Two test cases have been treated in order to show the efficiency of the procedure. First, an inductive coupling test case which is the TEAM Workshop problem no ${ }^{\circ}$. Second, a circuit coupling test case which is a laminated busbars, this last one being treated for a low frequency case and also for the DC case. Adaptive mesh refinement procedure has shown great potential and makes the unstructured PEEC method much more functional and attractive for modeling complex devices.
\end{abstract}

Index Terms-Integral Method, Unstructured Partial Element Equivalent Circuit (PEEC) method, Error Estimators, Adaptive mesh refinement.

\section{INTRODUCTION}

$\mathbf{P}$ OWER electrical devices are generally associated to complex geometries. The modeling of electromagnetic fields which is necessary in their design process can present errors, in particular with eddy current problems. Indeed, the accuracy of the numerical solution is crucial to correctly interpret the results obtained. The errors which can be induced are not only related to the numerical methods themselves, but also to a bad use of these methods. The most common is the sub-discretization of the domain which induces errors, especially for magneto-harmonic problems, where the correct representation of the electric current path is very important in order to accurately catch the skin effects. Numerical errors are also introduced with the use of a weak formulation including a material constitutive relationship. In this case, two physical quantities, which are continuous in two different functional spaces respectively, are only interpolated using a single functional space leading to local numerical inaccuracies.

The Partial Element Equivalent Circuit (PEEC) method [1], [2], [3] has been shown to be well suited for the analysis of many Electromagnetic (EM) devices, such as busbars, PCBs, integrated circuit interconnects, packaging and others. These devices have a rather plane geometry and their conductive parts are separated by a large amount of surrounding air, which is not necessary to mesh when using PEEC method, being an integral technique, unlike FEM methods where the air surrounding the devices must be well meshed. This characteristic represents one of the main advantages of PEEC, along with its ability to couple with electric circuits, thanks to the fact that PEEC formulation transforms Maxwell's equations on the active domain into an equivalent circuit representation.

The main motivation of this paper is to develop an efficient error estimator for the unstructured PEEC method, and to

Corresponding author: K. Alkama (email: kalkama@altair.com). couple it with an adaptive mesh refinement procedure. These developments will improve the accuracy without excessively increasing the number of degrees of freedom (DOF). This will make the method much more functional and attractive to model complex devices. Even if the PEEC method leads to dense matrices, there are matrix compression methods in order to reduce the computation time and memory consumption as much as possible. An Adaptive Multi Level Fast Multipole Method (AMLFMM) is used as the compression algorithm. This approach avoids the complexity of the parabolic calculation of the integration and resolution steps according to the DOF, but the complexity remains a major drawback of the PEEC method.

For the PEEC method, error estimators have not been investigated in the literature, but they have been widely studied for the finite element method (FEM). Two categories of error estimators have been developed. The first is called a priori error analysis and consists in evaluating the mesh quality according to the shape of its elements by detecting poor ones as shown in [4] and in [5]. This type of error analysis is not suitable for magneto-harmonic problems because it is entirely geometrical and supposes that the initial mesh already respects the skin depth, which is not generally the case. The second category is the a posteriori error analysis. It consists in evaluating the numerical solution quality using different estimator techniques. A quick and certainly non-exhaustive review of the different estimation techniques is presented in the introduction of the paper [6], and we can cite also [7] for superconvergent patch recovery (SPR) error estimator. A lot of work have been developed for magneto-harmonic FEM as shown in [8], [9], [10], but not yet for the unstructured PEEC method. In this paper, the a posteriori error analysis is originally focused.

The equilibrated error estimator [11] is maybe the most popular technique. It consists in comparing two complementary 
solutions of a problem obtained with two different formulations. For the eddy current problem, the technique starts from a first initial numerical solution $\mathbf{J}$ to build an admissible second $\mathbf{E}^{\prime}$ solution. It can be done by solving a dual formulation when available, or by projection and reconstruction using the appropriate tools as shown in [11]. This equilibrated error estimator is based on the non-verification of the material constitutive relationship (e.g. Ohm's law) in the active domain, using the initial and the admissible solutions.

The unstructured inductive PEEC method does not already have a clear dual formulation. To deal with the equilibrated estimator, an admissible solution is obtained by projecting the first initial solution and reconstructing it in the edge function space as shown in [11]. An energy-based error criterion approach has been used. It is supposed to be a good way to estimate the error in this case. This criterion is based on the accuracy on the computation of the energy stored in each mesh element, and it does not depend directly on the functional space of the solution, unlike the current density $\mathbf{J}$ or the electric field $\mathbf{E}$.

This paper is structured as follows. In Section III, the quasistatic (or inductive) unstructured PEEC formulation [12] is presented. Section III presents the method to compute the second admissible solution. Section IV] presents the method to compute the estimated error on the mesh. This error estimation will be compared to a reference error computed on a very fine mesh. Section $[\mathrm{V}$ presents the adaptive mesh refinement procedure which is coupled with the error estimator in order to refine the mesh where it is needed. Section VI presents some examples in order to validate the mesh refinement procedure. Finally a conclusion is given in Section VII

\section{UNSTRUCTURED INDUCTIVE PEEC FORMULATION}

Considering Maxwell's equations under quasi-static assumption with only non magnetic conductive material in a domain $\Omega$, the electric field $\mathbf{E}$ is:

$$
\mathbf{E}_{(P)}=-j \omega \mathbf{A}_{(P)}-\nabla \mathrm{V}_{(P)}
$$

where $\mathbf{A}$ is the magnetic vector potential, $\mathrm{V}$ is the electric scalar potential, $\omega$ is the angular frequency, and $P$ is the observation point. Considering $\mathbf{J}$ the current density, the magnetic vector potential $\mathbf{A}$ is

$$
\mathbf{A}_{(P)}=\frac{\mu_{0}}{4 \pi} \int_{\Omega} \frac{\mathbf{J}_{(Q)}}{r} d \Omega
$$

where $r$ is the distance between $P$ and the integration point $Q$, and $\mu_{0}$ is the magnetic permeability of the vacuum. Considering the following linear constitutive law

$$
\mathbf{J}=\sigma \cdot \mathbf{E}
$$

for $\Omega$ where $\sigma$ is the electrical conductivity, and introducing equations (2) and (3) in (1), we get

$$
\frac{\mathbf{J}_{(P)}}{\sigma_{(P)}}=-j \omega \frac{\mu_{0}}{4 \pi} \int_{\Omega} \frac{\mathbf{J}_{(Q)}}{r} d \Omega-\nabla V_{(P)}
$$

As proposed in [13], the current density $\mathbf{J}$ is interpolated with first-order face shape functions such as

$$
\mathbf{J}=\sum_{i} \mathbf{w}_{f_{i}} I_{i}
$$

where $\mathbf{w}_{\mathbf{f} i}$ is the face shape function and $I_{i}$ the electric current flowing through the face $i$. Applying a standard Galerkin projection procedure to (4) and using $\mathbf{w}$ as projection functions, a matrix system is obtained.

$$
\begin{gathered}
([\mathbf{R}]+j \omega[\mathbf{L}])\{I\}=\{\delta \mathrm{U}\} \\
\left\{\begin{array}{l}
{[\mathbf{R}]_{i, j}=\int_{\Omega} \frac{\mathbf{w}_{f_{i}} \mathbf{w}_{f_{j}}}{\sigma} d \Omega} \\
{[\mathbf{L}]_{i, j}=\frac{\mu_{0}}{4 \pi} \int_{\Omega} \mathbf{w}_{f_{i}} \int_{\Omega} \frac{\mathbf{w}_{f_{j}}}{r} d \Omega d \Omega} \\
\{\delta \mathrm{U}\}_{i}=-\int_{\Omega} \mathbf{w}_{f_{i}} \nabla V d \Omega
\end{array}\right.
\end{gathered}
$$

For the boundary faces of $\Omega$ not connected to the external circuit, the flowing current has to be imposed to zero using (8), where $\mathbf{n}$ is the external normal.

$$
\mathbf{J} \cdot \mathbf{n}=0
$$

Matrix system (6) can be seen as a classical circuit matrix system $\mathbf{Z I}=\mathbf{U}$, where each unstructured mesh element is associated to its equivalent circuit representation, as shown in Fig 1] This equivalent circuit is composed of one resistance
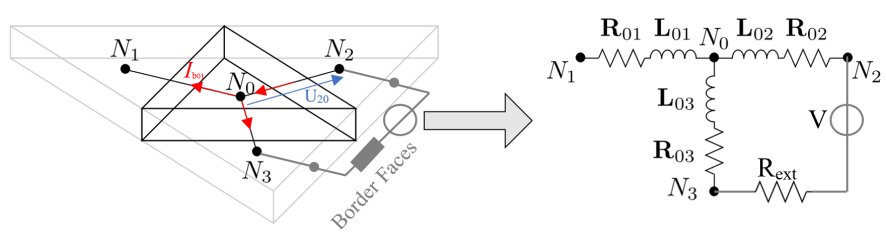

Fig. 1. Equivalent circuit representation for an unstructured mesh

and one self inductance for each face of the mesh element and is completed by all mutual inductances. This equivalent circuit can be solved using a classical circuit analysis approach in order to get all the electric quantities especially the electric current flowing in each branch which is an image of $\mathbf{J}$.

Note that formulations taking magnetic and dielectric materials are available [2], [3] for this work only the formulation with conductive material is used. In this paper, the solution $\mathbf{J}$ obtained by the equation (6) is considered as a primal solution. This solution has been found in the facets functional space associated to the mesh faces of the domain, this functional space naturally ensures the continuity of the normal component of J. Unfortunately, this functional space does not ensure the continuity of the tangential component of $\mathbf{E}$ which would be rather ensured with an edges functional space associated to the mesh edges of the domain.

\section{Computing the AdMissible SOlution $\mathbf{E}^{\prime}$}

In the literature, the unstructured PEEC method does not have actually a self dual formulation where the electric field $\mathbf{E}$ is the main unknown. So in order to calculate the admissible solution $\mathbf{E}^{\prime}$ which ensures the continuity of its tangential component in the domain $\Omega$, a projection and reconstruction 
method of $\mathbf{E}$ is used, as proposed in [11] but for the magnetic field $\mathbf{H}$.

In physics, the tangential component of the electric field $\mathbf{E}$ and the normal component of the current density $\mathbf{J}$ are continuous across the boundaries of the domain, they need also to be continuous for a discretization in edges and facets functional spaces respectively. Considering these properties, the current density obtained from the primal solution $\mathbf{J}$ can be scaled and projected from the facets to the edges functional space, this procedure is done by solving a finite element problem, according to:

$$
\sum_{j} \int_{\Omega_{e}} w_{e_{i}} \cdot w_{e_{j}} \cdot \mathbf{E}_{\mathbf{j}}^{\prime} d \Omega_{e}=\int_{\Omega_{e}} w_{e_{i}} \cdot \frac{\mathbf{J}}{\sigma} d \Omega_{e}
$$

where $w_{e}$ are the edge shape functions used for the interpolation, and $\frac{\mathbf{J}}{\sigma}$ represents values of the electric field $\mathbf{E}$ at Gauss points of each mesh element. The field $\mathbf{E}^{\prime}$ is obtained and the continuity of its tangential component on the mesh edges is ensured. This method is very efficient because a complete dual problem does not need to be solved, but only a FEM limited to the active material, i.e. very few DOF and a low memory need compared to PEEC approach to get the initial solution.

\section{CALCUlATION OF THE MESH DisCRETIZATION ERROR}

This section is divided in three subsections, the first one presents how to compute a reference error for a specific mesh in order to compare the error estimator presented in the second subsection, the comparison itself is done in the third subsection.

\section{A. Reference error for a mesh discretization}

Before trying to estimate a discretization error, a reference error is necessary to be able to validate error estimation. A rather intuitive method to calculate the reference error is proposed. Two meshes of the same simple parallelepipedic conductive volume are used. The first is meshed very coarsely and the other finely. This method is used very easily if the very fine reference mesh results from successive sufficient dichotomous divisions of the coarse mesh.

We aim to compute the error on the coarse mesh considering that the error is almost zero for the fine mesh. Let us suppose that a PEEC solution has been obtained for both meshes. The global error is written in the following equation:

$\varepsilon_{\text {global }}=\frac{\left|\int_{\Omega_{\text {coarse }}}\left(\mathbf{J}_{\text {coarse }}\right)^{2} d \Omega_{\text {coarse }}-\int_{\Omega_{\text {ref }}}\left(\mathbf{J}_{\text {ref }}\right)^{2} d \Omega_{\text {ref }}\right|}{\int_{\Omega_{\text {ref }}}\left(\mathbf{J}_{r e f}\right)^{2} d \Omega_{r e f}}$

where $\Omega_{\text {coarse }}$ and $\Omega_{\text {ref }}$ are the coarse and reference mesh global domains respectively, $\mathbf{J}_{\text {coarse }}$ and $\mathbf{J}_{r e f}$ are the current densities in the coarse and reference mesh respectively.

\section{B. Error estimation criterion}

Thanks to the methods presented in Section II and III, the primal $\mathbf{J}$ and the admissible $\mathbf{E}^{\prime}$ solutions are computed. The error in the discretization of the constitutive relation (3) can be estimated using the energy-based estimator [14]. The criterion is based on the computation of the difference of both values per mesh element of the energy, one computed using the primal solution and the other using the admissible one. This estimator allows to see clearly the computation error for the Joule losses.

In equation (11), the local error estimator is written in a normalized way according to a global quantity in the domain $\Omega$. This normalization helps to highlight the contribution of the local error of a mesh element to the global error.

$$
\eta_{\text {elem }}=N \times\left|\frac{\int_{\Omega_{\mathrm{E}}} \mathbf{J}^{2} d \Omega_{E}-\int_{\Omega_{\mathrm{E}}}\left(\sigma \cdot \mathbf{E}^{\prime}\right)^{2} d \Omega_{E}}{\int_{\Omega} \mathbf{J}^{2} d \Omega}\right|
$$

And the global error estimator is written as follows

$$
\eta_{\text {global }}=\left|\frac{\int_{\Omega} \mathbf{J}^{2} d \Omega-\int_{\Omega}\left(\sigma \cdot \mathbf{E}^{\prime}\right)^{2} d \Omega}{\int_{\Omega} \mathbf{J}^{2} d \Omega}\right|
$$

where $N$ is the number of mesh elements.

\section{Comparison between estimation and reference errors}

In this validation part, the simple mesh presented in Fig. 2 is used. It allows to compute the reference error easily using equation (10) and the regular hexahedral mesh with dichotomous divisions. A comparison is made for the behavior of global errors returned by the error estimator. The global error estimation calculated using equation (12) allows to evaluate the convergence of the global quantities.

In Fig. 2, a circular coil is fed by a current source with an intensity of $1 \mathrm{~A}$ at $500 \mathrm{~Hz}$. The 3D parallelepipedic plate is composed of copper. The dimensions are as follows:

- Circular non-meshed coil:

- Radius $30 \mathrm{~mm}$

- Cross-section $3.14 \mathrm{~mm}^{2}$

- 3D plate: $100 \times 100 \times 1 \mathrm{~mm}$

- Air-gap: $0.5 \mathrm{~mm}$ (very close)

The skin depth is equal to $3 \mathrm{~mm}$ for this case, the plate is overmeshed with 4 elements according to the thickness in order to ensure the quality of the solution in this direction. In theory, three elements in the skin depth with a first order interpolation are sufficient to well represent the variation of electric current. Therefore the dichotomous division only occurs in the plane parallel to the coil.

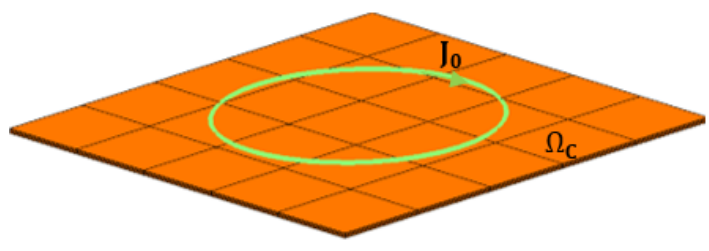

Fig. 2. Simple test case (3D plate under-meshed with a circular coil)

\section{1) Reference error computation}

In order to calculate the reference error, it is necessary to find a reference mesh which supposes that the solution obtained is sufficiently correct. Simulations are made on various refined meshes resulting from dichotomous divisions of the initial mesh presented in Fig. 2 The Joule losses in the plate are calculated for each mesh, this leads to determine the 
final one which is considered as the reference and associated to a null error. Fig. 3 shows the evolution of the Joule losses according to the number of elements, the losses starting to stabilize from the third mesh. The error between the fourth and the fifth mesh is negligible, this concludes that after four dichotomous cuts, a sufficient fine mesh is obtained to have correct results.

The last mesh is taken as reference, and then the errors on the other meshes are calculated, they are shown on the data labels in Fig. 3 .

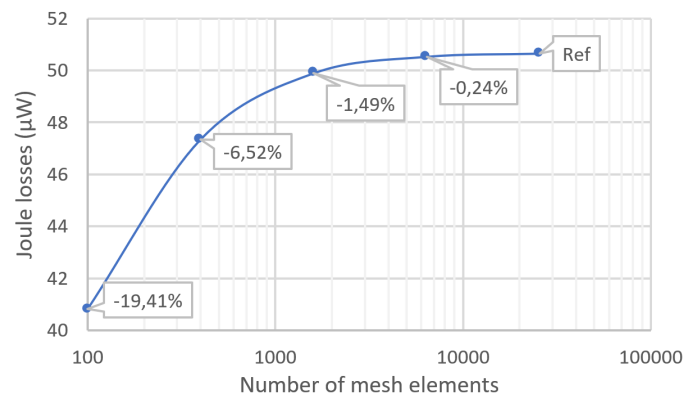

Fig. 3. Joule losses in the plate according to the number of mesh elements

The first mesh composed of 100 hexahedral elements is the worst among the other meshes in terms of under-meshing. We will try to estimate its numerical error with our error estimator.

\section{2) Error estimation}

In this part, the admissible solution $\mathbf{E}^{\prime}$ is considered. Fig. 4 presents the global errors estimated with the energy-based estimator between both solutions. These errors are calculated on the meshes introduced in the previous paragraph. Reference errors are also represented in Fig. 4. We can notice that estimated errors present a very good behavior compared to the right one.

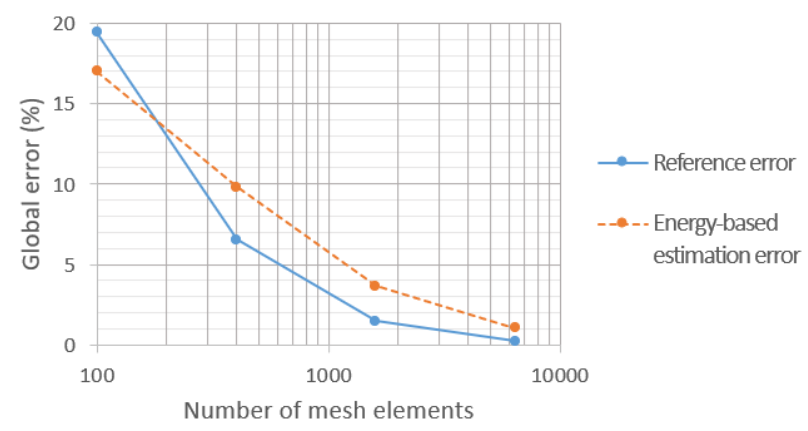

Fig. 4. Global errors evolution according to the number of mesh elements

It validates our PEEC-based error estimator, the edge projection method being fast with a complexity of approximately $\mathbf{O}(\mathrm{N})$, a good easiness in terms of implementation and without any modeling assumptions.

\section{ADAPTIVE MESH REFINEMENT PROCEDURE}

In order to test the error estimator, we will not use the prefinement strategy, i.e. we remain at the same interpolation order as in the equation (5). An adaptive refinement algorithm is developed using h-refinement, with a coupling with the mesh generator of the Altair Flux ${ }^{\text {тм }}$ FEM solver software [15]. The adaptive mesh refinement process has the following general structure:

1) Solve the problem with an initial coarse mesh that respects the geometry.

2) Estimate the local and the global error in the mesh.

3) If the estimated global error is acceptable, stop the process. Otherwise, elements that have local errors greater than the desired accuracy are selected for refinement.

4) Send the refinement information to the software, do the mesh refinement and return the new refined mesh.

5) Go to the first step and replace the previous mesh by the new refined one, and do iterations until the global error converges.

The mesh refinement procedure is executed, by taking a condition to refine an element via its local error estimation, such as

$$
\eta_{\text {local }_{\text {elem }}} \geq \frac{\eta_{\text {global }}}{2}
$$

where $\eta_{\text {local }_{\text {elem }}}$ is the local estimated error for a mesh element, and $\eta_{\text {global }}$ is the global estimated error.

Through this relative condition, our wish is to try to divide the estimated global error by two since it refines all the elements greater than its half, therefore where the errors are important at each iteration, and avoids dense random refinement.

The element cutting strategy consists in using only the surface mesh (the boundaries of the volume ). For each triangle element to be cut, a node is inserted in the middle of each edge among its three edges, thus four new triangles replace the initial triangle. By using this cutting strategy, we are obliged to also cut the neighboring triangles in half in order to keep the conformity of the mesh, as shown in Fig. 5, but currently without using any mesh regularization. Then the volume mesh is built using a classical Voronoi-Delaunay mesh generator taking the border mesh as a starting point.
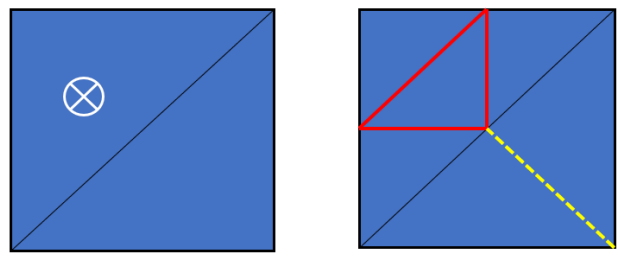

Fig. 5. Cutting strategy of a triangular element (regular division) and its neighbor (conformity division). Left: two triangles before cutting the one marked by a white cross. Right: cutting result, the new regular edges are in red, the conformity edge is yellow dashed

\section{APPLICATION EXAMPLES}

Several simulations are presented in this section. It includes pure eddy current problem as proposed in TEAM Workshop problem 7 [16] shown in Fig. 6] It includes also the simulation of a complex industrial test case with circuit type component connections, in DC but also with low frequency sources. This test case is a laminated bus bars. 


\section{A. TEAM Workshop problem 7}

The problem device is presented in Fig. 6. It consists of a coil and a volume plate with a hole. For the simulation, a nonmeshed coil powered by a 2742 A-turn real current source is selected. The plate is meshed with a initial tetrahedral conform mesh composed of 380 elements.

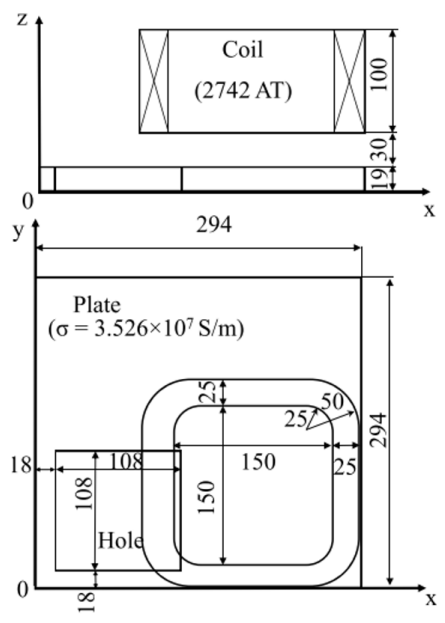

Fig. 6. Geometry of the TEAM problem 7 (the dimensions are in $\mathrm{mm}$ )

The proposed scenario consists in solving the problem at $50 \mathrm{~Hz}$ and at $200 \mathrm{~Hz}$, then in comparing the Joule losses computed with the references obtained in [13] with a dense FEM mesh of 2,000,000 elements. Let us notice this very fine FEM mesh has been chosen in order to be sure that the solution has converged and can be considered as a reference in order to determine the accuracy of the PEEC solution.

The adaptive mesh refinement procedure is executed in order to converge to a final mesh with a sufficiently low error. The evolution of Joule losses in the plate over the iterations of adaptive refinement is shown in Fig. 7) this figure also compares it with the reference results, and with those obtained with uniform refinement, i.e. all elements are cut out at each iteration. Table I presents a summary of the comparison between the results obtained over the adaptive refinement iterations with the FEM reference, and it also presents their cost in computation time.

TABLE I

RELATIVE ERROR OF THE PEEC ADAPTIVE REMESHING COMPARED WITH FEM SOLUTION AND THE TIME COSTS

\begin{tabular}{|l|l|l|l|l|l|l|l|l|}
\hline & $50 \mathrm{~Hz}$ & & & $200 \mathrm{~Hz}$ & & \\
\hline & Elements & $\begin{array}{l}\text { Joule } \\
\text { losses } \\
\text { (W) }\end{array}$ & Diff & $\begin{array}{l}\text { Time } \\
\text { cost } \\
\text { (min) }\end{array}$ & Elements & $\begin{array}{l}\text { Joule } \\
\text { losses } \\
\text { (W) }\end{array}$ & $\begin{array}{l}\text { Diff } \\
\begin{array}{l}\text { Time } \\
\text { cost } \\
\text { (min) }\end{array}\end{array}$ \\
\hline $\begin{array}{l}\text { FEM } \\
\text { 3D }\end{array}$ & $2 \mathrm{M}$ & 4.70 & Ref & $/$ & $2 \mathrm{M}$ & 9.5 & Ref & $/$ \\
\hline $\begin{array}{l}\text { PEEC } \\
2^{\text {nd }} \\
\text { mesh } \\
\text { iteration }\end{array}$ & 6,868 & 4.63 & $\begin{array}{l}1.48 \\
\%\end{array}$ & 2 & 4,629 & 8.92 & $\begin{array}{l}6.08 \\
\%\end{array}$ & 3 \\
\hline $\begin{array}{l}\text { PEEC } \\
3^{\text {rd }} \\
\text { mesh } \\
\text { iteration }\end{array}$ & 33,977 & 4.70 & 0.08 & 12 & 21,666 & 9.46 & 0.44 & 13 \\
$\%$ & & & & & & & & \\
\hline
\end{tabular}

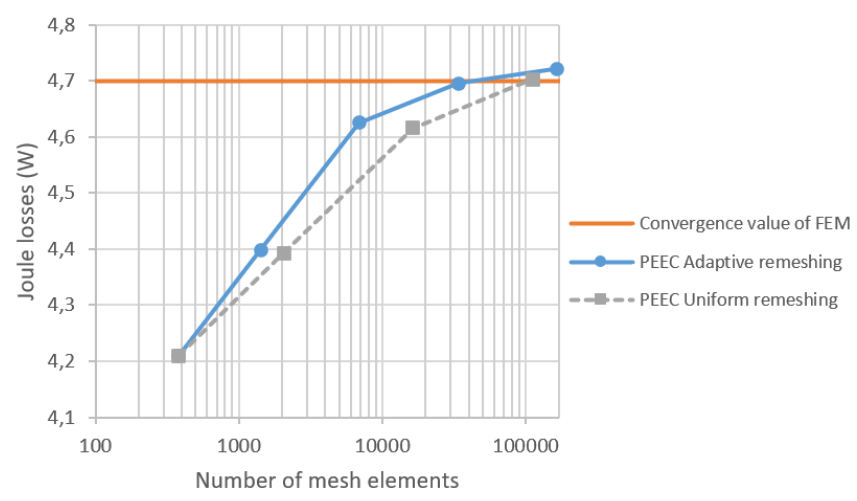

$(50 \mathrm{~Hz})$

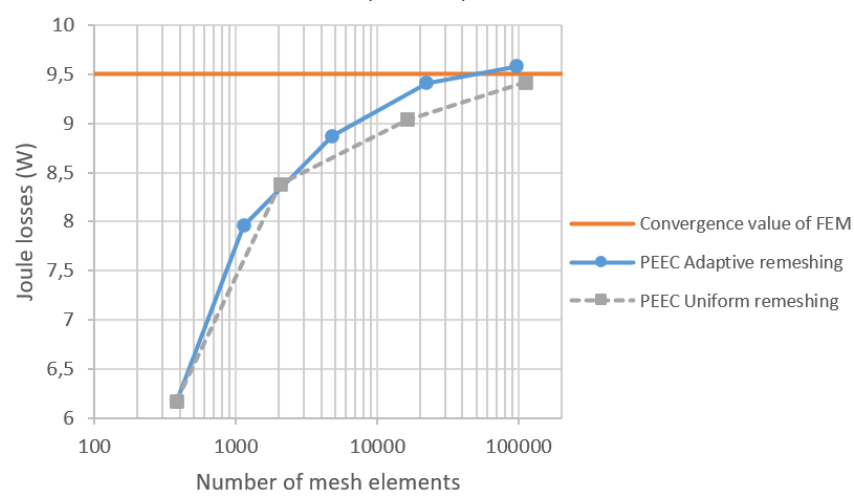

$(200 \mathrm{~Hz})$

Fig. 7. Joule losses values in the plate according to the number of mesh elements at $50 \mathrm{~Hz}$ and $200 \mathrm{~Hz}$

As shown in Fig7, we notice that the converged solution obtained with the FEM is quickly obtained and very close to the reference $(<6 \%)$, even if the starting mesh is very coarse as in this case. If we have a look at the uniform refinement procedure, the $4^{\text {th }}$ iteration could not be solved with our computer due to the large number of elements (around 800,000). In Fig. 7 at the $3^{\text {rd }}$ iteration, the same value of Joule loss is obtained for uniform and adaptive refinement, but with a large difference in terms of number of elements, as shown in Fig. 8 and in terms of computation time, $\mathbf{1 2}$ minutes for the adaptive and 103 minutes for the uniform from the start whole adaptive procedure $(50 \mathrm{~Hz})$. If we increase the frequency i.e increase of the skin effect or if we need to deal with a more complex geometry, basic uniform mesh refinement becomes unsustainable for the PEEC method making the adaptive mesh technique compulsory.

In Fig. 9. a comparison is done between the estimated global errors and real error, i.e. the Joule losses by taking the final mesh as a reference. The global estimated error has globally the same behavior compared to the real, which proves that the calculating method of the second admissible solution and the error estimator proposed are very efficient for dealing with this type of eddy current problem. The Fig. 10 presents a well distribution of the real part of the current density at $50 \mathrm{~Hz}$ and $200 \mathrm{~Hz}$.

The Fig. 11 shows the distribution of local errors estimated at $50 \mathrm{~Hz}$ for the initial and the final mesh. For the initial mesh, 


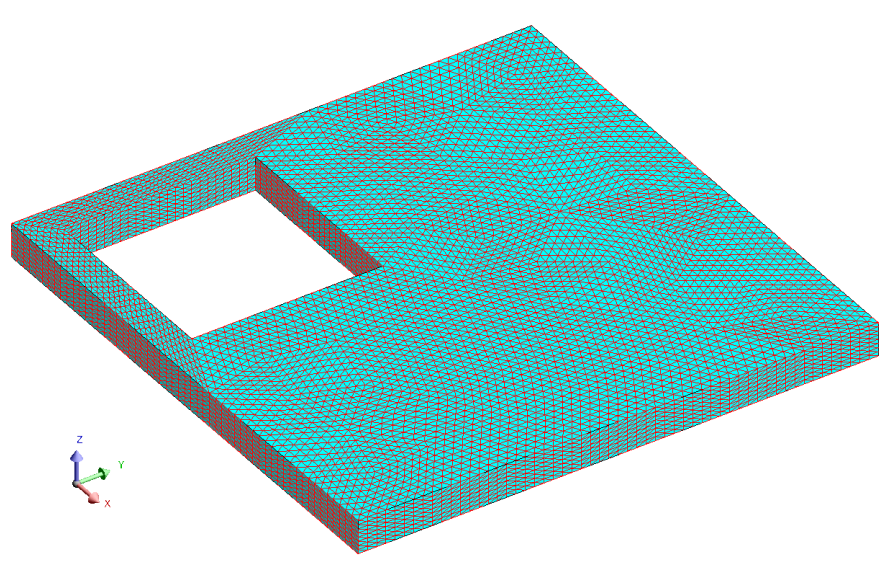

(a)

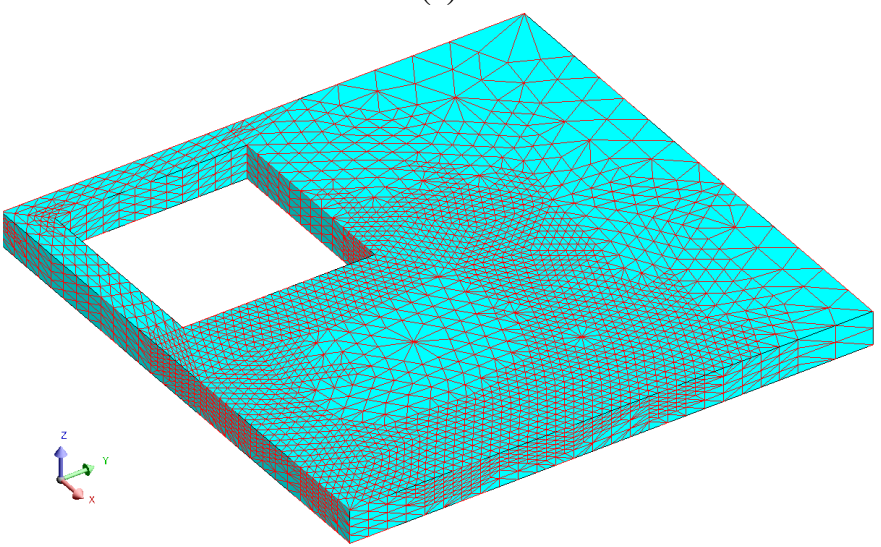

(b)

Fig. 8. The mesh obtained for the $3^{\text {rd }}$ iteration of refinement at $50 \mathrm{~Hz}$ : (a) uniform (112.000 elements), (b) adaptive (34.000 elements)

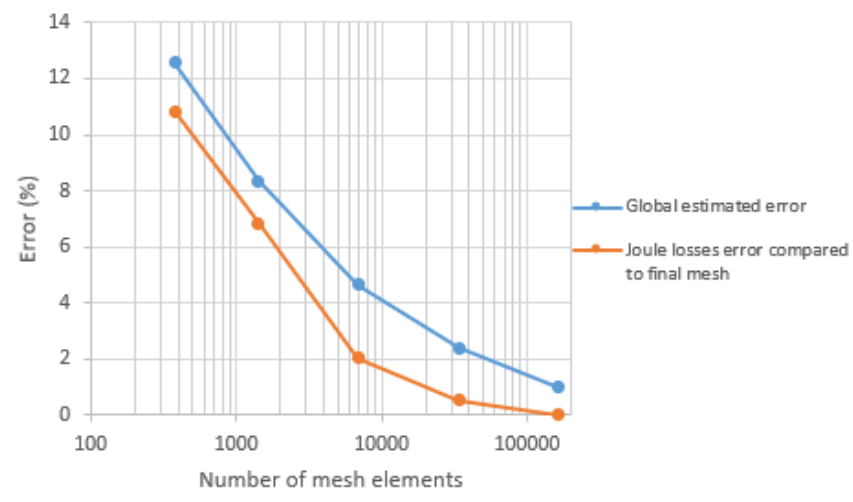

Fig. 9. Global errors on the plate at $50 \mathrm{~Hz}$

the local error seems to be higher on the main path of the eddy currents that must be refined. And for the final mesh, the error is considerably reduced except on very few elements located on the corner of the mesh where current density concentrates.

\section{B. Laminated busbars test case}

A problem with an industrial complexity has been solved. As shown in Fig. 12, it includes several connections with circuit type components (resistors, capacitors). The IGBT
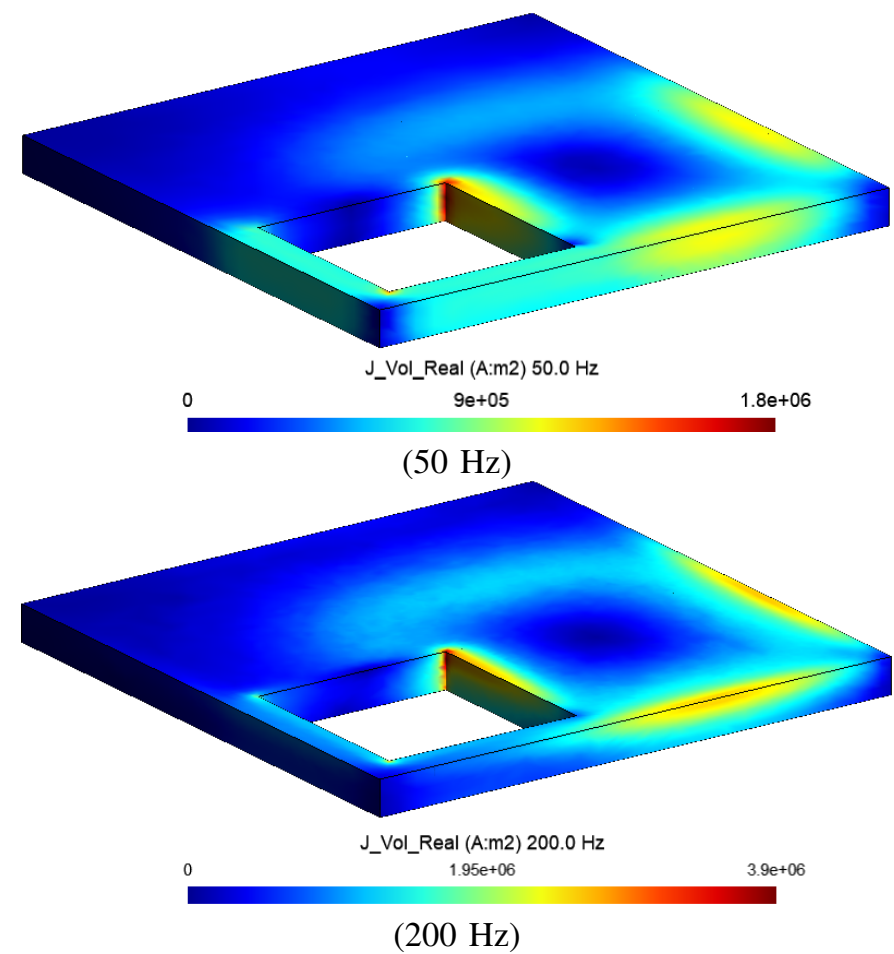

Fig. 10. Distribution of the Real part of the current density $\left(\mathrm{A} / \mathrm{m}^{2}\right)$ at $50 \mathrm{~Hz}$ and $200 \mathrm{~Hz}$

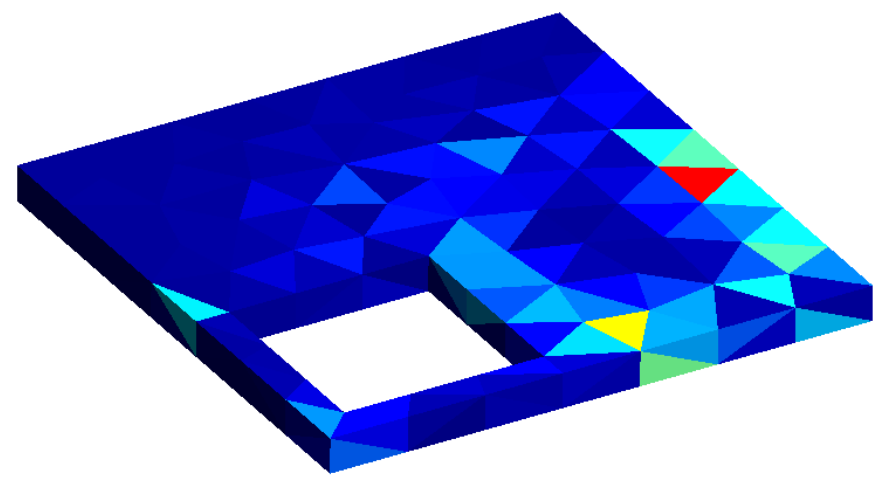

Estimated local error distribution $50 \mathrm{~Hz}$ (iteration 0 )

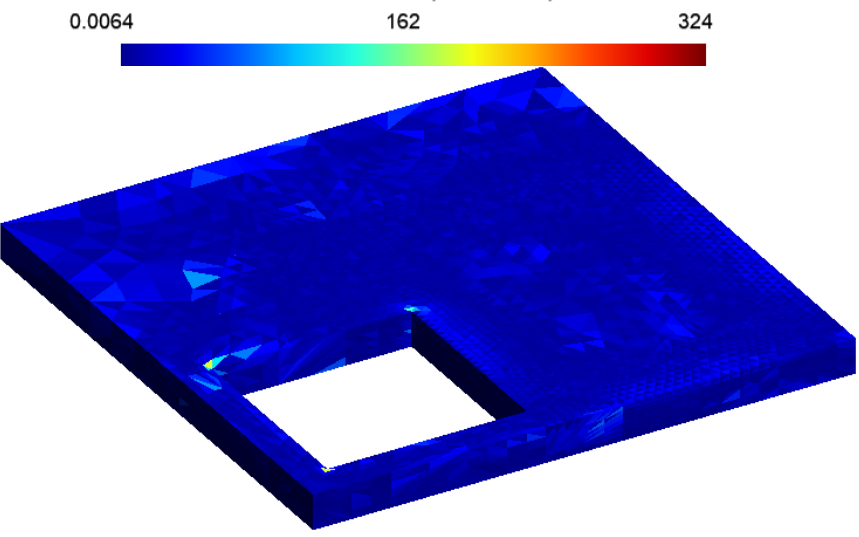

Estimated local error distribution $50 \mathrm{~Hz}$ (iteration 3) 0.000192

193 386

Fig. 11. Estimated local error distribution at $50 \mathrm{~Hz}$ for the initial and the final mesh 
and diodes have been modeled by their ON-state internal resistances. The circuit is fed by a $1 \mathrm{~V}$ voltage source.

In order to obtain the result shown in Fig. 12, considering the condition (13), a coarse initial mesh describing the geometry was considered. By choosing the global accuracy at $1 \%$, current distribution presented in Fig. 13 is obtained after 3 iterations of refinement procedure at $1 \mathrm{kHz}$. The global estimated error and the error on the Joule losses evolves rather in the same way over the iterations with a slight overestimation.

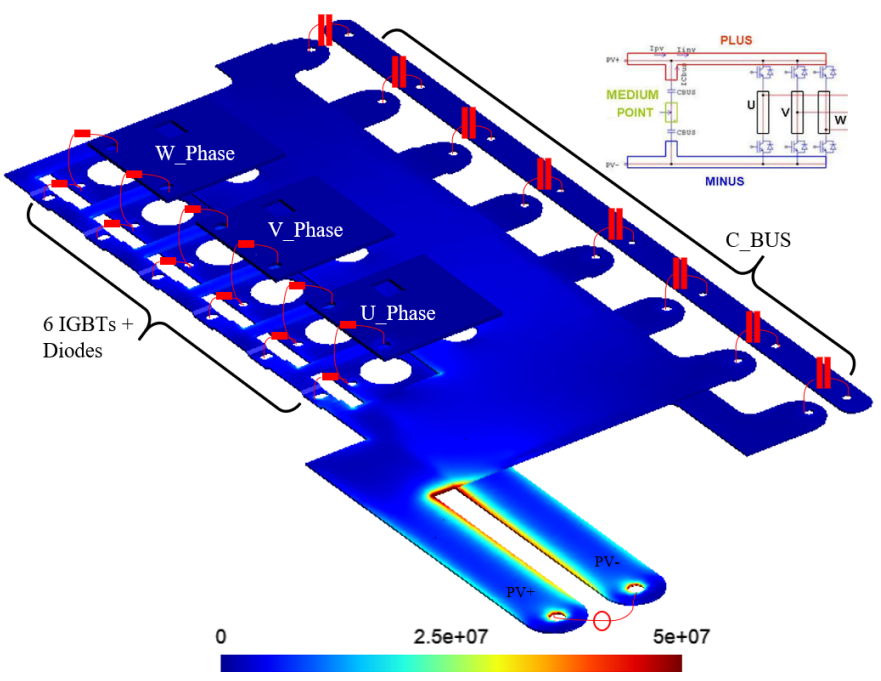

Fig. 12. Distribution of the current density $\left(\mathrm{A} / \mathrm{m}^{2}\right)$ on the laminated busbars when supplied by a voltage source of $1 \mathrm{~V}$ at $1 \mathrm{kHz}$

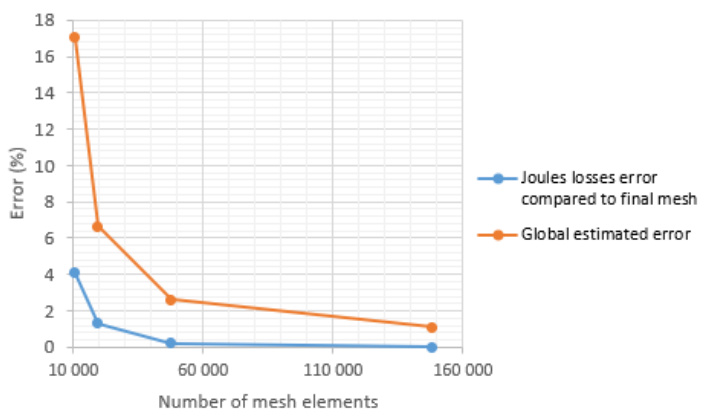

Fig. 13. Global errors on the laminated busbars at $1 \mathrm{kHz}$

For the DC case, the capacitors (C-BUS) in Fig. 12 have been replaced by resistors as shown in Fig. 14 In this case, although there is no skin effect, there are still numerical errors related to the sub-discretization of the geometry and of the electric current path. After two iterations of refinement procedure, we notice that the value of Joule losses does not really vary, this means that the initial mesh was sufficient enough to calculate the global quantities of the device as shown in Fig. 15, but the refinement has clearly improved the local quantities.

\section{CONCLUSION}

In this work, an error estimator has been proposed for the unstructured inductive PEEC based on two dual solutions.

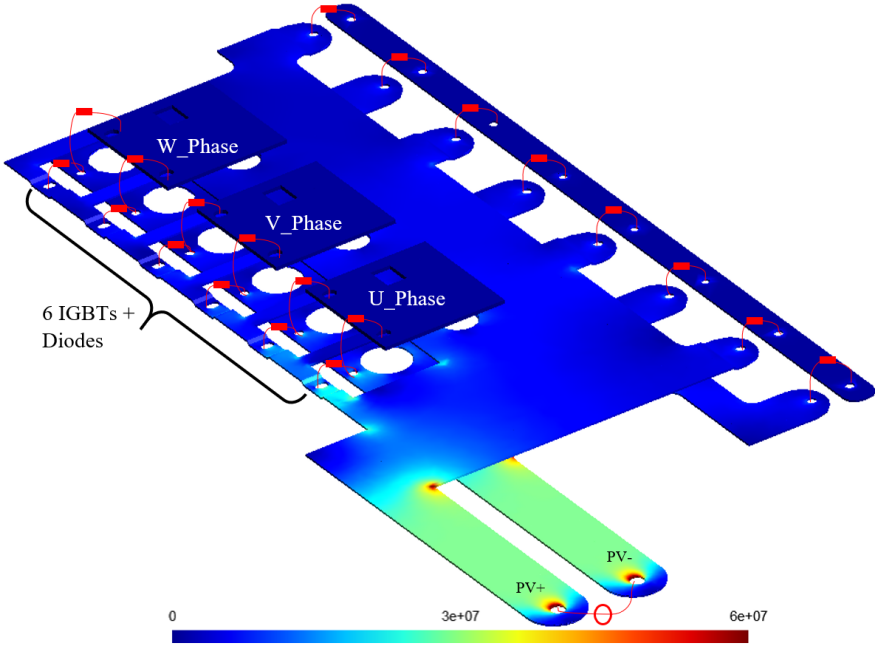

Fig. 14. Distribution of the current density $\left(\mathrm{A} / \mathrm{m}^{2}\right)$ on the laminated busbars when supplied by a voltage source of $1 \mathrm{~V}$ in DC

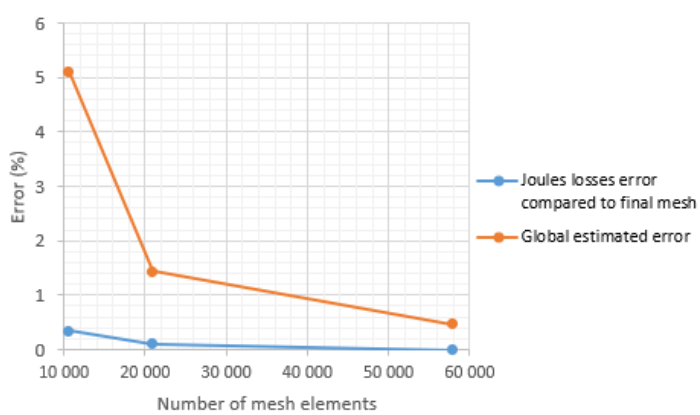

Fig. 15. Global errors on the laminated busbars in DC case

Avoiding to develop a dual formulation of PEEC method, an approach has been proposed to calculate a second admissible solution. An a posteriori error estimator has been used. It is based on the verification of the Ohm's constitutive relation in the mesh, this relation being weakly ensured by PEEC formulation. The estimator is an equilibrated energy-based criterion and has shown great efficiency. A method to calculate a so-called reference error on a regular hexahedral mesh has also been introduced, it made it possible to validate the error estimator.

Two test cases were handled. First, an inductive coupling test case which is the TEAM Workshop problem no ${ }^{\circ}$. It leads to very satisfactory results in comparison with a known solution obtained by FEM method. Second, a circuit coupling test case which is a laminated busbars, has been treated efficiently in low frequency case and in DC case. The adaptive mesh refinement procedure has shown great potential and makes the unstructured PEEC method much more functional and attractive for modeling complex devices.

\section{ACKNOWLEDGMENT}

We would like to thank David Pedreira and Delphine Dupuy R\&D Engineers at Altair Engineering for their help in setting up the mesh refinement part on the Altair Flux ${ }^{\text {тм }}$ software on which our refinement procedure is based. 


\section{REFERENCES}

[1] A. E. Ruehli, G. Antonini, J. Esch, J. Ekman, A. Mayo, and A. Orlandi, "Nonorthogonal PEEC formulation for time- and frequency-domain EM and circuit modeling," IEEE Trans. Electromagn. Compat., vol. 45, no. 2, pp. 167-176, 2003.

[2] R. Torchio, F. Moro, G. Meunier, J.-M. M. Guichon, and O. Chadebec, "An Extension of Unstructured-PEEC Method to Magnetic Media," IEEE Trans. Magn., vol. 55, no. 6, pp. 1-4, jun 2019.

[3] J. Siau, G. Meunier, O. Chadebec, J.-M. Guichon, and R. Perrin-Bit, "Volume Integral Formulation Using Face Elements for Electromagnetic Problem Considering Conductors and Dielectrics," IEEE Trans. Electromagn. Compat., vol. 58, no. 5, pp. 1587-1594, oct 2016.

[4] F.-X. Zgainski, Y. Marechal, J.-L. Coulomb, M. Vanti, and A. Raizer, "An a priori indicator of finite element quality based on the condition number of the stiffness matrix," IEEE Transactions on Magnetics, vol. 33, no. 2, pp. 1748-1751, mar 1997. [Online]. Available: http://ieeexplore.ieee.org/document/582611/

[5] J. R. Shewchuk, "What is a Good Linear Element ? Interpolation , Conditioning , and Quality Measures," Eleventh International Meshing Roundtable, pp. 115-126, 2002.

[6] L. Rondot, V. G. Mazauric, and P. F. Wendling, "An energy-compliant magnetodynamic error criterion for eddy-current calculations," IEEE Trans. Magn., vol. 46, no. 6, pp. 2353-2356, 2010.

[7] O. C. Zienkiewicz and J. Z. Zhu, "The superconvergent patch recovery (SPR) and adaptive finite element refinement," Comput. Methods Appl. Mech. Eng., vol. 101, no. 1-3, pp. 207-224, dec 1992. [Online]. Available: https://linkinghub.elsevier.com/retrieve/pii/ $004578259290023 \mathrm{D}$

[8] Z. Tang, Y. Le Ménach, E. Creusé, S. Nicaise, and F. Piriou, "Residual a posteriori estimator for magnetoharmonic potential formulations with global quantities for the source terms," IEEE Transactions on Magnetics, vol. 51, no. 3, pp. 15-18, 2015.

[9] E. Creusé, Y. Le Menach, S. Nicaise, F. Piriou, and R. Tittarelli, "Two guaranteed equilibrated error estimators for Harmonic formulations in eddy current problems," Computers \& Mathematics with Applications, vol. 77, no. 6, pp. 1549-1562, mar 2019. [Online]. Available: https://linkinghub.elsevier.com/retrieve/pii/S0898122118304723

[10] Z. Tang, S. Lou, A. Benabou, E. Creuse, S. Nicaise, J. Korecki, and J.-C. Mipo, "Guaranteed Quantity of Interest Error Estimate Based on Equilibrated Flux Reconstruction," IEEE Trans. Magn., vol. 9464, no. 1, pp. 1-1, 2021. [Online]. Available: https: //ieeexplore.ieee.org/document/9398695/

[11] J.-F. Remacle, C. Geuzaine, P. Dular, H. Hedia, and W. Legros, "Error estimation based on a new principle of projection and reconstruction," IEEE Transactions on Magnetics, vol. 34, no. 5, pp. 3264-3267, 1998. [Online]. Available: http://ieeexplore.ieee.org/document/717766/

[12] G. Meunier, O. Chadebec, J.-M. Guichon, V. Le-Van, J. Siau, B. Bannwarth, and F. Sirois, "A-T Volume Integral Formulations for Solving Electromagnetic Problems in the Frequency," IEEE Trans. Magn., vol. 52, no. 3, pp. 1-4, mar 2016.

[13] T.-T. Nguyen, G. Meunier, J.-M. Guichon, O. Chadebec, and T.-S. Nguyen, "An Integral Formulation for the Computation of 3-D Eddy Current Using Facet Elements," IEEE Transactions on Magnetics, vol. 50, no. 2, pp. 549-552, feb 2014. [Online]. Available: http://ieeexplore.ieee.org/document/6749118/

[14] D. Ladas, V. Mazauric, G. Meunier, O. Chadebec, M. Ebene-Ebene, Y. Maréchal, and P. Wendling, "An energy based approach of electromagnetism applied to adaptive meshing and error criteria," IEEE Trans. Magn., vol. 44, no. 6, pp. 1246-1249, 2008.

[15] Altair Engineering, "Altair Flux ${ }^{\mathrm{TM}}, "$ 2020. [Online]. Available: https://www.altair.com/flux/

[16] L. R. Turner, K. Davey, N. Ida, D. Rodger, A. Kameari, A. Bossavit, and C. R. I. Emson, "Workshops and problems for benchmarking eddy current codes," United States, Tech. Rep., 1988. [Online]. Available: http://inis.iaea.org/search/search.aspx?orig \{_\}q=RN:20009769 